\title{
Genetic Analysis of Avian Influenza Viruses: Cocirculation of Avian Influenza Viruses with Allele A and B Nonstructural Gene in Northern Pintail (Anas acuta) Ducks Wintering in Japan
}

\author{
Alam Jahangir, ${ }^{1,2}$ Sakchai Ruenphet, ${ }^{1,3}$ Nadia Sultana, ${ }^{1}$ \\ Dany Shoham, ${ }^{1,4}$ and Kazuaki Takehara ${ }^{1,3}$ \\ ${ }^{1}$ Laboratory of Zoonoses, School of Veterinary Medicine, Kitasato University, Towada 034-8628, Japan \\ ${ }^{2}$ Animal Health Research Division, Bangladesh Livestock Research Institute, Savar, Dhaka 1341, Bangladesh \\ ${ }^{3}$ Tokyo University of Agriculture and Technology, Tokyo 183-0057, Japan \\ ${ }^{4}$ Begin-Sadat Center for Strategic Studies, Bar-Ilan University 52900, Ramat Gan, Israel
}

Correspondence should be addressed to Alam Jahangir, alamjahan2003@yahoo.com

Received 21 September 2012; Revised 2 December 2012; Accepted 6 December 2012

Academic Editor: Zichria Zakay-Rones

Copyright (C) 2012 Alam Jahangir et al. This is an open access article distributed under the Creative Commons Attribution License, which permits unrestricted use, distribution, and reproduction in any medium, provided the original work is properly cited.

\begin{abstract}
The pandemic influenza virus strains of 1918 (H1N1), 1957 (H2N2), 1968 (H3N2), and 2009 (H1N1) have genes related to avian influenza viruses (AIVs). The nonstructural (NS) gene of AIVs plays a significant role in host-viral interaction. However, little is known about the degree of diversity of this gene in Northern pintail (Anas acuta) ducks wintering in Japan. This study describes characteristics of pintail-originated H1N1, H1N2, H1N3, H5N2, H5N3, H5N9, and H7N7 viruses. Most of the viruses were revealed to be avian strains and not related to pandemic and seasonal flu strains. Nevertheless, the NP genes of $62.5 \%$ (5/8) viruses were found closely related to a $\mathrm{A} /$ swine/Korea/C12/08, indicating exchange of genetic material and ongoing mammalianlinked evolution of AIVs. Besides, all the viruses, except Aomori/422/07 H1N1, contain PSIQSR*GLF motif usually found in avian, porcine, and human $\mathrm{H} 1$ strains. The Aomori/422/07 H1N1 has a PSVQSR* GLF motif identical to a North American strain. This findings linked to an important intercontinental, Asian-American biogeographical interface. Phylogenetically all the viruses were clustered in Eurasian lineage. Cocirculation of allele A and B (NS gene) viruses was evident in the study implying the existence of a wide reservoir of influenza A viruses in pintail wintering in Japan.
\end{abstract}

\section{Introduction}

Influenza A virus infections in birds account for important inputs into the evolutionary porcine-human complex of this prominent anthropozoonotic pathogen. Among influenza A viruses, which broadly exhibit 17 HA and 9 NA antigenic subtypes, only three haemagglutinin (HA) subtypes (H1, H2, and H3) and two neuraminidase (NA) subtypes (N1 and N2) have circulated widely in swine and human populations since the 20th century [1]. Viruses from waterfowl reassorted with existing human and/or porcine influenza viruses to generate the 1957, 1968 [2], and 2009 (Novel swine-origin influenza A (H1N1) virus investigation team, 2009) pandemic influenza viruses and may expectably play a similar role in the creation of future pandemic viruses. In addition, on multiple occasions, it has been evident that avian influenza viruses (AIVs), chiefly the subtypes H5N1, H7N7, and H9N2, directly transmitted from birds to humans $[3,4]$. Avianoriginated $\mathrm{H} 1 \mathrm{~N} 1, \mathrm{H} 3 \mathrm{~N} 2, \mathrm{H} 5 \mathrm{~N} 1$, and $\mathrm{H} 9 \mathrm{~N} 2$ viruses have been recovered from pigs in Asia, Europe, and Canada [57]. Furthermore, $\mathrm{H} 2 \mathrm{~N} 3$ avian virus reassortants were isolated from pigs in the United States [8].

Pigs have been postulated, hence, to be the ultimate "mixing vessels" for mammalian influenza viruses and AIVs and can play an important role in the genetic reassortment of influenza viruses. Therefore, detection and characterization of AIVs, especially those subtypes having the potential to transmit to mammals, including pigs and humans, are significant. Avian H7N7 and H3N8 strains are contracted and circulated by horses, as well, and an equine $\mathrm{H} 3 \mathrm{~N} 8$ virus 
apparently was involved in the formation of the pandemic $\mathrm{H} 3 \mathrm{~N} 2$ virus, and perhaps the H3N8 pandemic virus of 1889 [9].

The genome of influenza A viruses consists of eight different segments of single-stranded negative-sense RNA. Most gene segments encode for one protein; whereas the gene segments 2 (polymerase basic1-PB1), 7 (matrix$\mathrm{M}$ ), and 8 (nonstructural-NS gene), each encodes one additional protein from alternatively spliced mRNA. Among the proteins, 9 are structural, while the rest two, namely PB1-F2 and NS1, are nonstructural. Thirteen genes have already been identified all over. Aquatic birds are the primary reservoir of type A influenza viruses and are classified into various antigenic subtypes, based on their two surface glycoproteins, the HA, and the NA. So far, seventeen HA (H1H17) and nine NA subtypes (N1-N9) have been identified from avian species [10], representing the entire pool of influenza A viruses known today. Most of them are not found or uncommon within mammalian hosts. Although largely shaped by the HA, the virulence of influenza A viruses is polygenic in nature. $\mathrm{H} 5$ and $\mathrm{H} 7$ influenza viruses with multiple basic amino acids nearby to the cleavage site of the HA glycoprotein exhibit a wide range of tissue tropism and lead to systemic disease in chickens with fatality [11].

It has been demonstrated that the amino acids at positions 627 and 701 of the polymerase basic 2 (PB2) protein influence the outcome of infection in mice $[12,13]$. Several studies have reported that the NS1 protein is also associated with the virulence of influenza viruses $[14,15]$. The glutamic acid at position 92 of NS1 of H5N1 influenza virus confers virulence and resistance to antiviral cytokines in pigs [16]. In spite of those well-known, meaningful functions of the NS gene concerning virulence and escape from host cytokine response, the degree of variation in the NS gene pool of AIVs in their natural reservoirs, particularly in the Northern pintail (Anas acuta) (thereafter referred to as pintail (s)) ducks, a globally major influenza host wintering in Japan, is poorly studied. In this paper, we focused on genetic analysis of $\mathrm{H} 1 \mathrm{~N} 1, \mathrm{H} 1 \mathrm{~N} 2$, and H1N3 strains of AIVs that we isolated from apparently healthy migratory pintails wintering in Japan $[17,18]$. Also, we analyzed the NS genes of $\mathrm{H} 5$ and $\mathrm{H} 7$ subtypes, in addition to the above-mentioned AIVs. Moreover, polymerase acid (PA), nucleoprotein (NP), $\mathrm{M}$, and NS genes of H5N9 strain, an uncommon antigenic combination which we isolate for the first time in Asia [19], were further looked into in the present study.

\section{Material and Methods}

2.1. Viruses. Viruses used in this study (Table 1) were of low pathogenicity and isolated in embryonated chicken eggs (ECE) from fecal materials of migratory, apparently healthy pintails wintering in Japan $[17,18]$. Working stocks of viruses were prepared by 3 rd passage in ECE, and allantoic fluid was harvested at 3 days after inoculation (dpi) and stored at $-80^{\circ} \mathrm{C}$.

2.2. RNA Extraction and RT-PCR. Total viral RNA was extracted from infected allantoic fluid using the Isogen-LS
(Nippongene, Tokyo, Japan), in accordance with the manufacturer's instructions. Reverse transcription was carried out with the Uni12 primer (5'-AGC AAA AGC AAG G$3^{\prime}$ ) and MMLV reverse transcriptase (GeneAmp RNA PCR Kit, Applied Biosystems, Tokyo, Japan), followed by full length amplification of each gene segment, as described earlier [20]. The PCR product was purified using QIAquick Gel Extraction Kit (Qiagen, Valencia, CA), according to the manufacturer's instructions.

2.3. Gene Sequencing. The PCR product was sequenced by dideoxy chain terminating method, using Dye Terminator Cycle Sequencing FS Ready Reaction Kit (Perkin-Elmer Applied Biosystems, Japan). Sequencing was carried out with the same primers used to amplify the gene and continued with subsequently designed sequence-specific primers. Nucleotide sequences were determined using an automated DNA sequencer (ABI 310 DNA sequencer, Applied Biosystems, Foster City, CA), edited and assembled with GENETYX-Mac (version 10.0; Software Development Corp., Tokyo, Japan).

2.4. Sequence and Phylogenetic Analysis. Multiple sequence alignments and processing were performed with the Molecular Evolutionary Genetics Analysis (MEGA) version 4.1.0 software, with an engine based on the ClustalW algorithm [21]. Blast searches were used to retrieve the homologous sequences from the GenBank database. The phylogenetic analyses were performed using neighbor joining tree inference analysis, with the 1000 bootstrap replications, to assign confidence levels to branches. Nucleotide sequences of entire open reading frame (ORF) of each gene segment (except PB1 gene of A/northern pintail/Aomori/1130/08 H1N3 (Aomori/1130/08 H1N3)) were used in the phylogenetic analysis. The PB1 gene of Aomori/1130/08 H1N3 was partially 1350 base pair (1-1350 bp) sequenced and used in the phylogenetic analysis. Furthermore, the HA gene of A/northern pintail/Akita/1364/08 H1N2 (Akita/1364/08 H1N2) was sequenced partially 765 bp (760-1524) and was not included in the phylogenetic analysis.

2.5. GenBank Accession Number. The nucleotide sequences generated in this study have been deposited in GenBank and are available under accession number AB546149-AB546193.

\section{Results and Discussion}

As a part of longitudinal virological studies, we have isolated various subtypes of AIVs from fecal materials of migratory, apparently healthy pintails wintering in Tohoku district, Japan $[17,18]$. Of these, H1N1, H1N2, and H1N3 viruses were characterized genetically. For phylogenetic relationships of NS gene, the latter from viruses bearing $\mathrm{H} 1, \mathrm{H} 5$, and H7 HA was sequenced and compared with viruses from GenBank.

3.1. Genetic and Phylogenetic Analysis of HA Genes. The sequenced HA genes $(n=6)$ of $\mathrm{H} 1$ subtype with different NA subtype combinations $(\mathrm{N} 1, \mathrm{~N} 2$, and N3) isolated from 


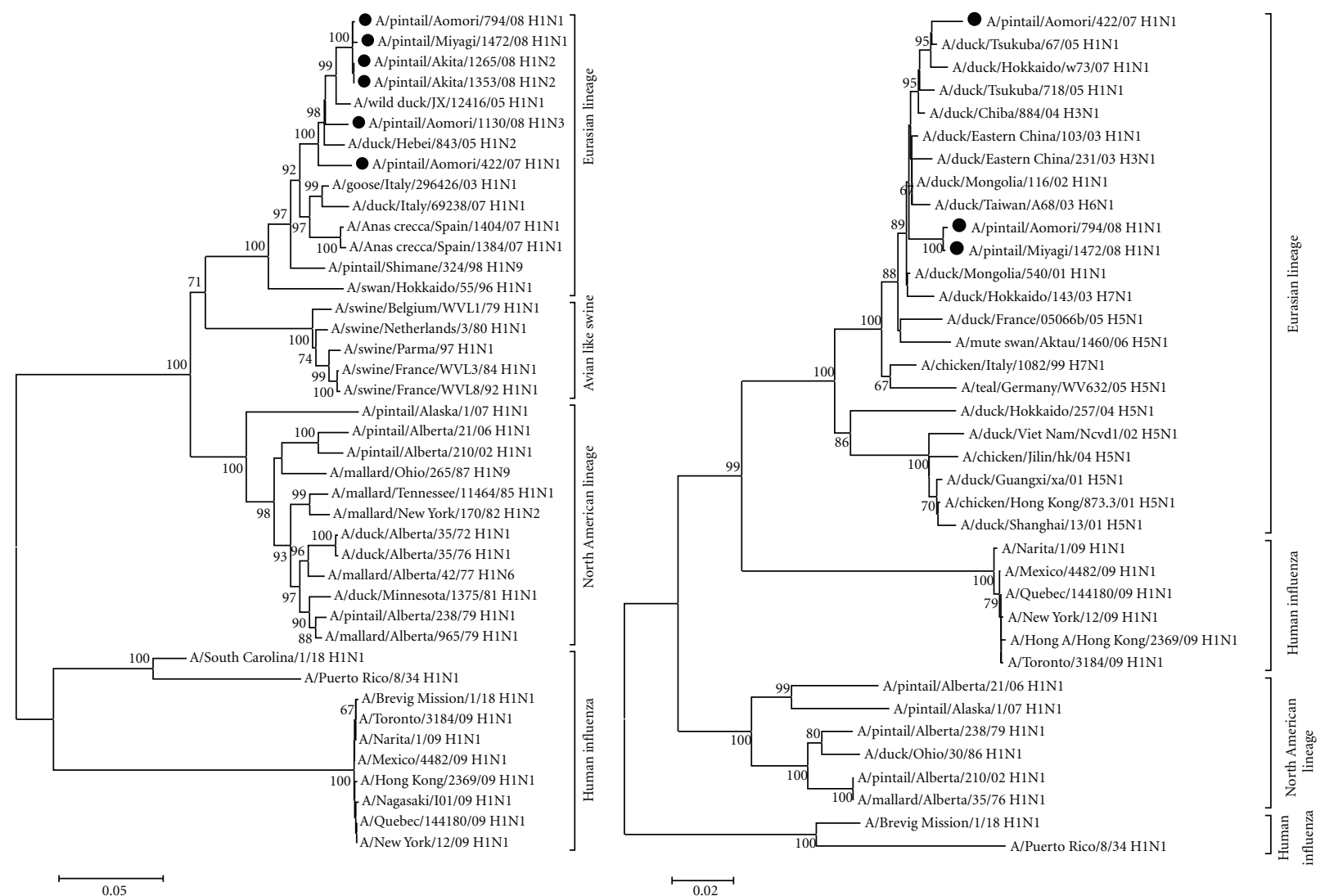

(a)

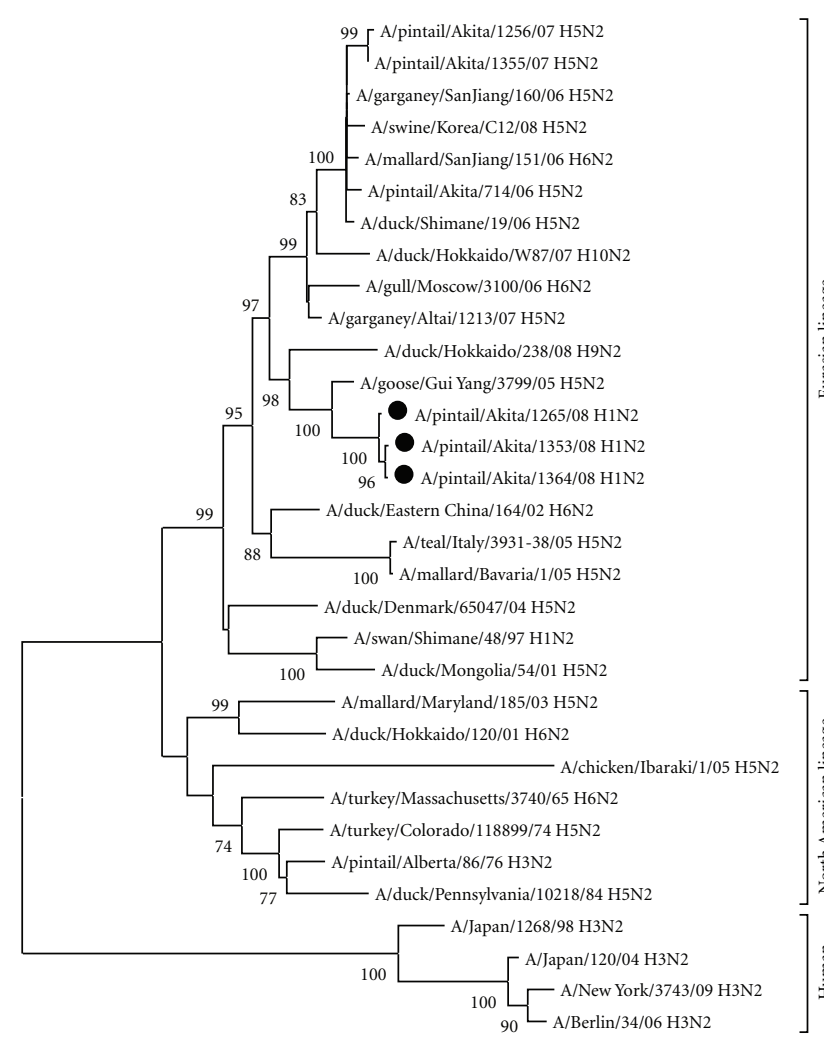

$\longmapsto 0.02$
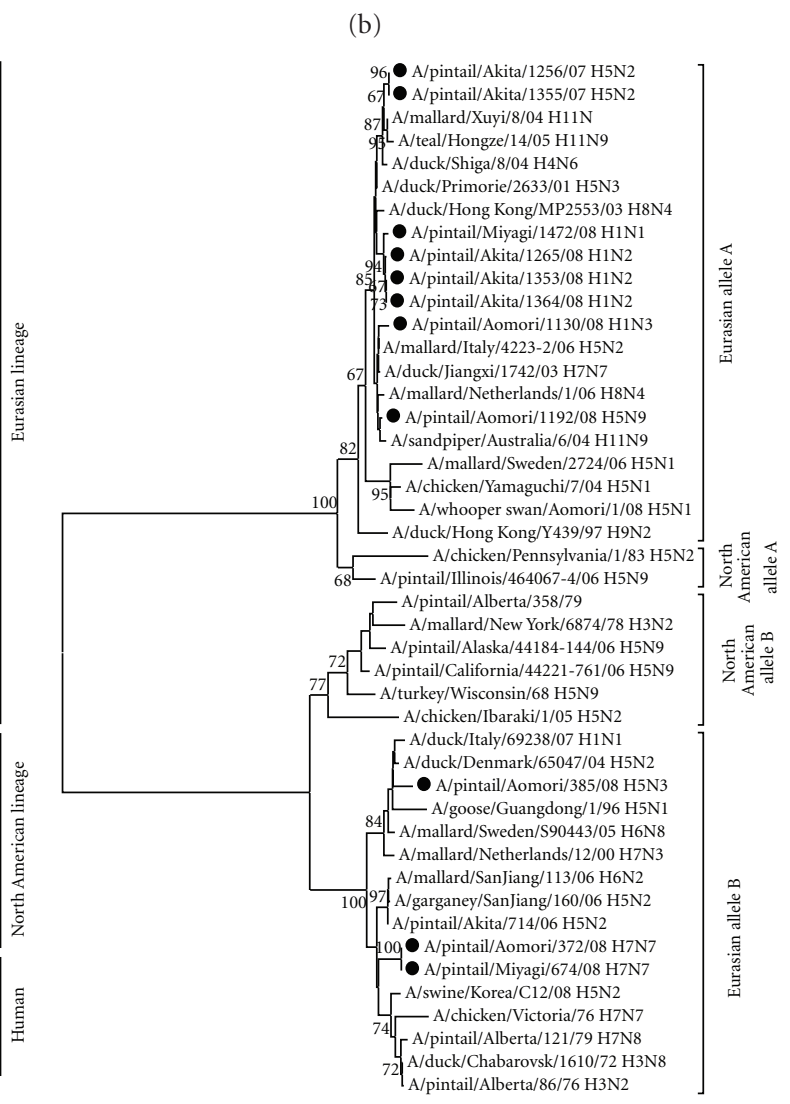

(c)

(d)

Figure 1: Continued. 


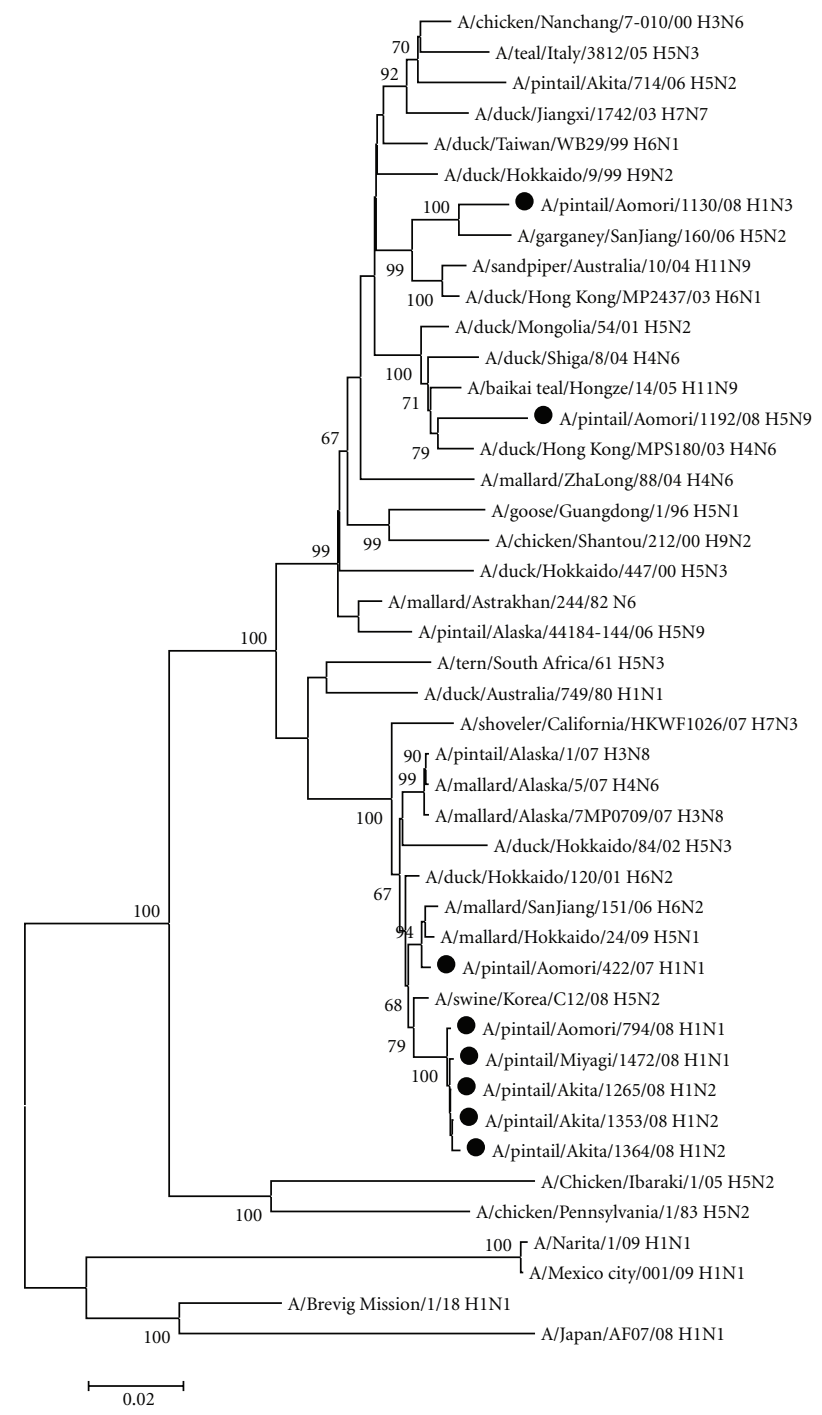

(e)

Figure 1: Phylogenetic analysis of avian influenza viruses isolated from Northern pintails in Tohoku district, Japan. Included are different segment of AIVs. Individual tree was generated using neighbor-joining method and 1,000 replications of bootstrap resampling. The number at each branch point indicates percentage probability that the resultant topology is correct. The ORF of each gene segment (a) H1 (1701 bp), (b) N1 (1410 bp), (c) N2 (1410 bp), (d) NS (838 bp), and (e) NP (1497 bp) was employed to generate phylogram. Viruses of the present study were marked by black circle $(\bullet)$ in the tree. Strains with no host were assumed to human isolates.

pintails during 2007-2008 were closely related to each other (Figure 1(a); Table 2). The nucleotide and amino acid sequence identities were found to range from 96.5 to $100 \%$ and 98.0 to $100 \%$, respectively. Phylogenetically, the viruses were clustered with the viruses from Eurasian countries, mostly with viruses from China, namely A/duck/Hebei/843/05 H1N2 and A/wild duck/JX/12416/05 H1N1 (>97\% nucleotide homology) (Figure 1(a)). The HA genes of sequenced viruses were grouped into the h1.1.2 (subtype H1) [22]. Viruses of the present study had a sister group relationship with the Japanese $\mathrm{H} 1$ viruses including A/pintail/Shimane/324/98 H1N9 isolated earlier. North American strains, and swine, human seasonal and pandemic $\mathrm{H} 1$ strains integrated sharply in different branches. It means that although bearing the same antigenic subtype (H1N1) and obtained during 2007-2008, our isolates were unrelated to the porcine-derived 2009 pandemic and seasonal flu strains.

3.2. Genetic and Phylogenetic Analysis of NA Genes. Like HA genes, the nucleotide homologies of the NA genes of the isolates with each other were $96.8-99.8 \%$ and $99.7-99.9 \%$ for $\mathrm{N} 1(n=3)$ and N2 $(n=3)$, respectively, for each subtype. On the other hand, the amino acid sequence identities were found to range from 97.4 to $99.8 \%(\mathrm{~N} 1)$ and $99.4-99.8 \%$ (N2). A duck-originated Japanese strain obtained 2 years earlier, A/duck/Tsukuba/67/05 H1N1 (Tsukuba/67/05 H1N1) 
TABLE 1: Avian influenza viruses used in the present study ${ }^{\mathrm{a}}$.

\begin{tabular}{|c|c|c|}
\hline Virus & Gene segment sequenced & $\begin{array}{l}\text { HA0 cleavage site } \\
\left({ }^{*} \text { cleavage point }\right)^{\mathrm{b}}\end{array}$ \\
\hline A/pintail/Aomori/422/07 H1N1 & $\mathrm{HA}, \mathrm{NP}, \mathrm{NA}$, and $\mathrm{M}$ & PSVQSR*GLF \\
\hline A/pintail/Aomori/794/08 H1N1 & $\mathrm{HA}, \mathrm{NP}, \mathrm{NA}$, and $\mathrm{M}$ & PSIQSR*GLF \\
\hline A/pintail/Miyagi/1472/08 H1N1 & $\mathrm{HA}, \mathrm{NP}, \mathrm{NA}, \mathrm{M}$, and NS & PSIQSR*GLF \\
\hline A/pintail/Akita/1265/08 H1N2 & $\mathrm{HA}, \mathrm{NP}, \mathrm{NA}, \mathrm{M}$, and NS & PSIQSR*GLF \\
\hline A/pintail/Akita/1353/08 H1N2 & $\mathrm{HA}, \mathrm{NP}, \mathrm{NA}, \mathrm{M}$, and NS & PSIQSR* GLF \\
\hline A/pintail/Akita/1364/08 H1N2 & $\mathrm{HA}^{\mathrm{c}}, \mathrm{NP}, \mathrm{NA}, \mathrm{M}$, and NS & PSIQSR* GLF \\
\hline A/pintail/Aomori/1130/08 H1N3 & $\mathrm{PB} 2, \mathrm{~PB}^{\mathrm{c}}, \mathrm{PA}, \mathrm{HA}, \mathrm{NP}, \mathrm{NA}, \mathrm{M}$, and NS & PSIQSR*GLF \\
\hline A/pintail/Aomori/1192/08 H5N9 & $\mathrm{PA}, \mathrm{NP}, \mathrm{M}$, and NS & $-\mathrm{d}$ \\
\hline A/pintail/Akita/1256/07 H5N2 & NS & - \\
\hline A/pintail/Akita/1355/07 H5N2 & NS & - \\
\hline A/pintail/Aomori/385/08 H5N3 & NS & - \\
\hline A/pintail/Aomori/372/08 H7N7 & NS & - \\
\hline A/pintail/Miyagi/674/08 H7N7 & NS & - \\
\hline
\end{tabular}

Table 2: Percentage identity (\%) of nucleotide sequences of the HA genes of avian influenza viruses: intrahomology and interhomology with interrelated sequences from GenBank.

\begin{tabular}{lcl}
\hline Virus & Identity $^{\mathrm{a}}(\%)$ & Highest identity $^{\mathrm{b}}(\%)$ \\
\hline Subtype H1 & & A/duck/Hebei/843/05 H1N2 (97.2) \\
A/pintail/Aomori/422/07 H1N1 & & \\
A/pintail/Aomori/794/08 H1N1 & & \\
A/pintail/Miyagi/1472/08 H1N1 & $96.5-100$ & A/wild duck/JX/12416/05 H1N1 (>97.3) \\
A/pintail/Akita/1265/08 H1N2 & & \\
A/pintail/Akita/1353/08 H1N2 & & \\
A/pintail/Aomori/1130/08 H1N3 & &
\end{tabular}

${ }^{\mathrm{a}}$ Nucleotide sequence identity among the isolates; ${ }^{\mathrm{b}}$ identity with genes of viruses from GenBank.

was found most closely related to one of the isolatesA/northern pintail/Aomori/422/07 H1N1 (Aomori/422/07 H1N1) (>98\% homology at both nucleotide and amino acid sequences)—of the present study, while two other viruses have maximum homology to a Mongolian strain, A/duck/Mongolia/116/02 H1N1 (Mongolia/116/02 H1N1), isolated in 2002 (>98\% homology at both nucleotide and amino acid sequences). For N2 NAs, a Chinese strain, namely A/goose/Gui Yang/3799/05 H5N2 (Gui Yang/3799/05 H5N2) isolated from goose in 2005 was found rather closely related ( $>98 \%$ nucleotide and amino acid identity). The N3 NA gene of Aomori/1130/08 H1N3 has $>99 \%$ nucleotide and amino acid homology to a duck strain, namely A/duck/Niigata/514/06 H5N3 (Niigata/514/06 H5N3) isolated in 2006 in Japan (Table 3). Principally, the NA genes of the sequenced viruses were clustered with viruses of Eurasian origin (Figures 1(b) and 1(c)). More specifically they can be grouped into sublineages n1.1.8 (N1 subtype) and n2.1.6 (subtype N2) [22]. NA gene of Aomori/422/07 $\mathrm{H} 1 \mathrm{~N} 1$ strain branched separately from other strains isolated by us in 2008 (Figure 1(b)), the N2 NA genes of which were all integrated into a single branch represented by a H5N2 strain, Gui Yang/3799/05, which was isolated in China in 2005 (Figure 1(c)). Like H1 HA genes, the N1 NA genes were distinctly separated from pandemic strains (Figure 1(b)).

3.3. Genetic and Phylogenetic Analysis of NS Genes. A remarkably diverse variation in the nucleotide sequence identities (50-100\%) was found among the NS genes of the sequenced viruses (Table 4 ). When analyzed phylogenetically, they were distinctly branched into two branches, allele A and B [23], and clustered with Eurasian origin viruses (Figure 1(d) and Table 4). Nucleotide sequence identities of NS genes within allele A and within allele B were $97.7-100 \%$ and $94.4-100 \%$, respectively. However, the divergence between the two alleles was approximately 50\%, regardless of their isolation time and geographical location. The majority of the viruses of the present study were branched with allele $\mathrm{A}$ viruses represented with/by A/mallard/Sweden/2724/06 H5N1 and A/duck/Hong Kong/Y439/97 H9N2 (Hong Kong/Y439/97 H9N2). In contrast, A/northern pintail/Aomori/372/08 H7N7 (Aomori/372/08 H7N7), Aomori/385/08 H5N3, and A/northern pintail/Miyagi/674/08 H7N7 (Miyagi/674/08 
TABLE 3: Percentage identity (\%) of nucleotide sequences of the NA genes of avian influenza viruses: intrahomology and interhomology with interrelated sequences from GenBank.

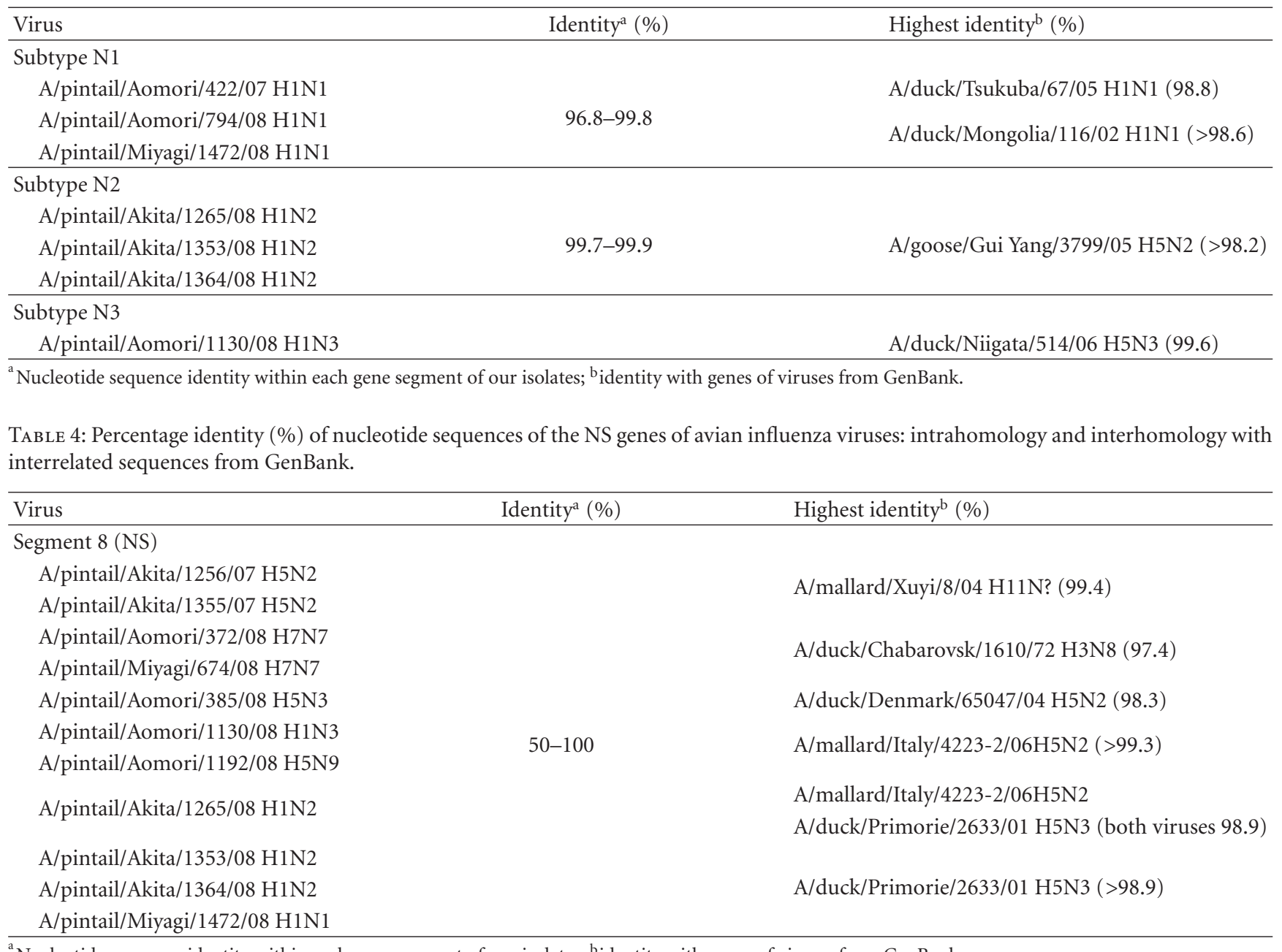

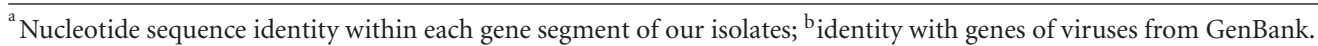

H7N7) viruses were clustered with allele $B$ viruses represented with/by A/goose/Guangdong/1/96 H5N1 (Guangdong/1/96 H5N1). As for allele A, two pairs-Akita/1256/ 07 H5N2 and Akita/1355/07 H5N2, alongside with Akita/ 1353/08 H1N2 and Akita/1364/08 H1N2 viruses-were found 100\% homologous, while in allele B Aomori/372/08 H7N7 and Miyagi/674/08 H7N7 viruses were found to be entirely identical.

Significantly, the NS1 genes of influenza viruses in general have been divided into two alleles: $\mathrm{A}$ and $\mathrm{B}$ [23]. Regardless of some exceptions, all influenza viruses circulating in mammalian species and many viruses from avian species comprise the allele A, while allele B is found within only AIVs. The NS1 gene of the majority of highly pathogenic avian influenza (HPAI) $\mathrm{H} 5 \mathrm{~N} 1$ viruses isolated from humans since 1997 is affiliated with allele A. In contrast, the NS1 gene of the Guangdong/1/96 virus, which was the source of the initial HPAI H5N1/1997 HA gene, belonged to allele B [24]. The NS1 genes of viruses of the present study-all LPAI-belong either to allele A or allele B. Curiously, it was pointed out that some strains of the
HPAI H5N1 virus were preserved genetically unchanged from 1997 up to 2005 [25]. In that connection, perhaps, it is of note that the NS gene of some viruses, for example Aomori/372/08 H7N7 and Miyagi/674/08 H7N7 of this study have only $<3 \%$ nucleotide disparity from that of a Russian strain, namely A/duck/Chabarovsk/1610/72 H3N8 (Chabarovsk/1610/72 H3N8), isolated in 1972. The NS gene of one of the viruses we had isolated from pintail ducks, namely Akita/714/06 H5N2, has 98.3\% nucleotide homology to the same Russian isolate Chabarovsk/1610/72 H3N8 [26]. It means that the ongoing mutation rate during about 35 years was only about $1.7 \%$, which is markedly less than the expected one. This may imply that throughout about a third of that 35 years period the NS gene has been somehow conserved, considering that the estimated NS gene evolutionary rates for avian, classic swine, and equine lineages are $0.84-1.27$ nucleotide changes per site per year [27], and even higher, within both wild and domestic avian host species [28]. Basically, the two reasons thought to possibly account for unexplained genetic conservation in influenza A viruses are cross contamination, or interference 
with vaccine strains [29]. The strain Chabarovsk/1610/72 or related strains are not held in our laboratory, and interference with a vaccine strain seems unlikely, in that case. Abiotic environmental virus preservation has been suggested as an alternative explanation [30]. These, together with the location of Chabarovsk being close to the regular migration route of pintails between Japan and Siberia, have to further be looked into.

Prevalence of allele A and B viruses in their natural hosts has been reported to vary spatially, as well. Generally, allele $\mathrm{B}$ viruses were reported to be less common in their natural hosts than allele A. Out of 11 NS genes sequenced in this study, $72.7 \%(8 / 11)$ were classified as allele $\mathrm{A}$, and $27.3 \%(3 / 11)$ as allele B. In Asia, the prevalence of allele $B$ viruses in all avian species has been reported to be only $15 \%$ [31], which is markedly lower than found in this study. However, the prevalence rate of allele B viruses in North American free flying birds has been documented to be $30 \%$, similar to that observed in pintails in our study [32], but higher than found in Northern European mallards (13\%; 6 out of 45) [31]. This might be due to the migration pattern of pintails sampled in our study. Most pintails which winter in Japan originate from eastern Russia [33, 34]. Besides, pintails marked in North America have been recovered during winter in Japan and vice versa $[35,36]$. Moreover, some pintails migrate from North America to eastern Russia [37], where they could come into contact with birds that migrate from six continents, including Asian wintering sites [38]. Furthermore, from the recent satellite telemetry data, provided by the United States Geological Survey (USGS) and the US Fish and Wildlife Service, it is evident that pintails marked during winter in Japan move to Alaska through Russia and return to Japan, following the same route (Alaska Science Center, Movements of Northern Pintail ducks and Whooper Swans marked with satellite transmitters in Japan http://alaska.usgs.gov/science/biology/ avian_influenza/pintail_movements_virus.php. Thus, interand intra continental exchange of genes and genomes of AIV could occur through congregation of North American and Asian migrants at shared summer habitats in eastern Russia, or by means of pintails that migrate between North American nesting grounds and Japanese wintering grounds (assessment of virus movement across continents: using Northern Pintails (Anas acuta) as a test. http://alaska.usgs.gov/science/biology/avian_ in-fluenza/migration_ecology.php. Also the number of samples may possibly have effect on the results found in this study.

3.4. Genetic and Phylogenetic Analysis of NP Genes. The NP genes of the sequenced viruses have $90.4-99.9 \%$ nucleotide homologies among each other, regardless of their temporal and spatial differences in isolation (Table 5). Broadly, viruses isolated from Eastern Hemisphere have high homologies to the NP genes of the viruses of this study (97.2 to $99.5 \%$ nucleotide identity). It is noteworthy that the NP gene of the majority of viruses ( $75 \%$ of 8 viruses) investigated in this study had a high homology to a porcine strain, namely A/swine/Korea/C12/08 H5N2 (Korea/C12/08 H5N2) isolated in 2008, suggesting that the NP genes of these viruses and the Korea isolate might have a common ancestral origin. Similar to NP gene of $\mathrm{H} 1$ viruses, the HA genes of some H5N2 viruses isolated by us during 2006-2007 were found to be closely related to this Korean virus $[19,26]$. In conjunction, biogeographically and phylogenetically, other related strains, namely A/garganey/San Jiang/160/06 H5N2, A/baikal teal/Hongze/14/05 H11N9, and A/duck/Hong Kong/MPS180/03 H4N6 originated from China, and one strain, A/mallard/Hokkaido/24/09 H5N1, from Japan. For NP gene viruses from both Eurasian countries and North America were clustered with the viruses of the present study (Figure 1(e)). The nucleotide sequence disparity between viruses of this study and North American viruses clustered together ranged from 1.4 to $9.3 \%$. Especially, and not by chance, in all likelihood, Aomori/422/07 H1N1 has 98.6\% nucleotide identity to the strain A/pintail/Alaska/1/07 H3N8, meaning isolated from the same host in the same year. In that case, viruses of North American lineage integrated distinctly from those of Eurasian lineage, suggesting, in light of the mentioned high NP gene homology, intercontinental gene exchange through reassortment, rather than whole genome intercontinental conveyance. Such interfaces are at any rate significant, evolutionarily and epidemiologically.

3.5. Molecular Features of HA and NA. The HA amino acid sequences deduced from nucleotide sequences analysis revealed that all the viruses contain seven potential $\mathrm{N}$-linked glycosylation sites (5 in HA1 and 2 in HA2) throughout the HA molecule. Except for Aomori/422/07 H1N1, the amino acid motif of the HA0 cleavage site of all the sequenced $\mathrm{H} 1$ viruses was PSIQSR*GLF (*cleavage point), which is common in all avian, porcine, and human viruses. Aomori/422/07 H1N1 contains a rare amino acid motif, PSVQSR* GLF. Among the $\mathrm{H} 1$ avian strains isolated globally and sequences reported in GenBank during 1980-2009, only one strain, namely A/mallard/New York/170/82 H1N2 has identical (PSVQSR*GLF) amino acids. However, Aomori/422/07 H1N1 solely clustered with the Eurasian viruses (Figure 1(a)), implying, ostensibly that it has no relation with the mallard-originated New York strain and evolves individually. Still, the extreme rareness of the mentioned motif might possibly be supportive of genetic recombination event that perhaps took place within collocated ducks from North America and Asia. The HA molecules of all of the sequenced $\mathrm{H} 1$ viruses contain residues Gln $(\mathrm{Q})$ and Gly (G) at positions 240 and 242 (H1 numbering), respectively, which indicate the avian receptor specificity.

On the other hand, the amino acid sequence analysis of NAs revealed that all the viruses contain full length NA protein with seven potential $\mathrm{N}$-linked glycosylation sites in $\mathrm{N} 1$ and N2 known to be conserved in wild ducks [39]. In all NA molecules, a residue His $(\mathrm{H})$ was found at position 274 (N2 numbering), thereby indicating sensitivity to Oseltamivir or Zamamivir [40]. Besides, typical catalytic sites, framework sites as well as other specific regions were found completely conserved as described previously [41]. Residue position 198 in N3 NA contains Asn (N) instead of Asp (D) and is also shown to be conserved [41]. The N1 and N2 NA of 
TABLE 5: Percentage identity (\%) of nucleotide sequences of nucleoprotein (NP) coding genes of avian influenza viruses: intrahomology and interhomology with interrelated sequences from GenBank.

\begin{tabular}{|c|c|c|}
\hline Virus & Identity $^{\mathrm{a}}(\%)$ & Highest identity $^{\mathrm{b}}(\%)$ \\
\hline \multicolumn{3}{|l|}{ Segment 5 (NP) } \\
\hline \multirow{2}{*}{ A/pintail/Aomori/422/07 H1N1 } & & A/garganey/SanJiang/160/06 H5N2 \\
\hline & & A/mallard/Hokkaido/24/09 H5N1 (both viruses 99.5) \\
\hline \multicolumn{3}{|l|}{ A/pintail/Aomori/794/08 H1N1 } \\
\hline \multicolumn{3}{|l|}{ A/pintail/Akita/1265/08 H1N2 } \\
\hline A/pintail/Akita/1353/08 H1N2 & $90.4-99.9$ & A/swine/Korea/C12/08 H5N2 (>98.8) \\
\hline \multicolumn{3}{|l|}{ A/pintail/Akita/1364/08 H1N2 } \\
\hline \multicolumn{3}{|l|}{ A/pintail/Miyagi/1472/08 H1N1 } \\
\hline A/pintail/Aomori/1130/08 H1N3 & & A/garganey/SanJiang/160/06 H5N2 (97.7) \\
\hline A/pintail/Aomori/1192/08 H5N9 & & $\begin{array}{l}\text { A/baikal teal/Hongze/14/05 H11N9 A/duck/Hong Kong/MPS180/03 H4N6 } \\
\text { (both viruses 97.2) }\end{array}$ \\
\hline
\end{tabular}

${ }^{a}$ Nucleotide sequence identity within each gene segment of our isolates; ${ }^{b}$ identity with genes of viruses from GenBank.

the sequenced viruses possessed E119 and R292 amino acid residues, indicating that the commonly mutated residues in NA inhibitor-resistant viruses were not present [42].

3.6. Molecular Features of NS1. Deletion of several amino acids in the NS1 gene has been observed more frequently in AIVs in recent years, a feature of possible adaptation of these viruses to poultry $[24,43]$. The viruses sequenced in our study contained full length NS genes, indicating basically wild bird strains. Nevertheless, all isolates possessed residue Ala (A) position 149, which is important for replication of viruses in chickens [24]. Also, residue Asp (D) instead of Glu (E) was found at position 92 of the NS1, which is reported to be involved in modulation of cytokine response, and has been associated with the high virulence exhibited by HPAI $\mathrm{H} 5 \mathrm{~N} 1$ viruses in pigs [16]. All the sequenced viruses contain a PDZ (postsynaptic density, PSD-95; discs large, Dlg; zonula occludens-1, ZOO-1) domain ligand at the C terminus of NS1 (ESEV-COOH), which plays an important role in many key signaling pathways of viral replication [44].

3.7. Relation between Avian H1N1 Strains and Pandemic and Seasonal Influenza Strains. When compared to pandemic and seasonal influenza viruses, the HA genes' nucleotide identity of viruses of the present study was found to range from 67.1 to $76.4 \%$, while amino acid sequence homology was found $81.8-92.1 \%$ (Table 6). In difference, for NA gene, a somewhat higher nucleotide and amino acid identity was found (79.3-86.5\% nucleotide and 84.9-92.5\% amino acid sequence identities). Not only the HA and NA genes but also other internal genes of pandemic and seasonal flu strains were clustered distinctly from those of our isolates, suggesting no relation between these viruses. Basically, genomic analyses of the last four pandemic strains showed that the genes contributed by avian strains are those that encode for the polymerases and surface antigens, as follows: 1918-PB1 and PA; 1957-PB1, HA, and NA; 1968-PB1 and HA; 2009_-PB2 and PA. This means that among the presently prevailing pandemic and seasonal strains, only
TABle 6: Comparison of avian H1N1 strain with pandemic and seasonal flu strains.

\begin{tabular}{lccc}
\hline \multirow{2}{*}{ Virus } & \multicolumn{3}{c}{ Aomori/422/07 H1N1 identity (\%) } \\
& Nucleotide & $\begin{array}{c}\text { Amino } \\
\text { acid }\end{array}$ & Nucleotide \\
\hline Segment 4 (HA) & & & \\
A/Brevig Mission/1/18 H1N1 & 67.8 & 92.1 & 67.1 \\
A/Mexico city/001/09 H1N1 & 75.3 & 82.5 & 74.7 \\
A/Japan/AF07/08 H1N1 & 76.4 & 83.6 & 76.4 \\
\hline Segment 6 (NA) & & & \\
A/Brevig Mission/1/18 H1N1 & 84.5 & 92.3 & 84.5 \\
A/Mexico city/001/09 H1N1 & 86.5 & 90.0 & 86.5 \\
A/Japan/AF07/08 H1N1 & 79.3 & 84.9 & 79.5 \\
\hline
\end{tabular}

the seasonal $\mathrm{H} 3 \mathrm{~N} 2$ has past affinity to avian HA, when it originally formed as a then pandemic strain in 1968, thereafter considerably drifting genetically for already 43 years. Independent drift probably took place during that period of time within the precursor avian $\mathrm{H} 3 \mathrm{HA}$ gene in bird populations.

\section{Conclusion}

In conclusion, our genetic analysis suggests that the sequenced viruses were to an appreciable degree characteristic of the pintail populations we sampled, which typically winter in Japan. Except in one strain, the HA0 cleavage site of $\mathrm{H} 1$ was found as usually found in all avian, porcine, and human viruses. The residues that compose the catalytic and framework sites of the NA enzyme were completely conserved in the studied viruses. The NP gene of the majority of the strains sequenced in this study $(5 / 8)$ was related to that of the porcine strain Korea/C12/08 H5N2. Thus, it may be presumed that our isolates evolved through reassortment process during the cocirculation of these strains. Our findings clearly demonstrate that two distinct gene pools, corresponding to both NS allele A and B, were present within 
the pintail populations wintering in Japan. It is noteworthy that some strains contain NS gene highly related to a duck isolate obtained in Russian in 1972, about 35 years prior to our isolate, indicating significant gene conservation. It is also concluded that although bearing the same antigenic subtype (H1N1) and obtained during 2007-2008, our isolates were unrelated to 2009 pandemic and seasonal flu strains.

\section{References}

[1] L. Campitelli, C. Fabiani, S. Puzelli et al., "H3N2 influenza viruses from domestic chickens in Italy: an increasing role for chickens in the ecology of influenza?" Journal of General Virology, vol. 83, no. 2, pp. 413-420, 2002.

[2] Y. Kawaoka, S. Krauss, and R. G. Webster, "Avian-to-human transmission of the PB1 gene of influenza A viruses in the 1957 and 1968 pandemics," Journal of Virology, vol. 63, no. 11, pp. 4603-4608, 1989.

[3] E. C. J. Claas, A. D. M. E. Osterhaus, R. Van Beek et al., "Human influenza A H5N1 virus related to a highly pathogenic avian influenza virus," Lancet, vol. 351, no. 9101, pp. 472-477, 1998.

[4] Y. P. Lin, M. Shaw, V. Gregory et al., "Avian-to-human transmission of H9N2 subtype influenza A viruses: relationship between H9N2 and H5N1 human isolates," Proceedings of the National Academy of Sciences of the United States of America, vol. 97, no. 17, pp. 9654-9658, 2000.

[5] Y. Guan, K. F. Shortridge, S. Krauss, P. H. Li, Y. Kawaoka, and R. G. Webster, "Emergence of avian H1N1 influenza viruses in pigs in China," Journal of Virology, vol. 70, no. 11, pp. 80418046, 1996.

[6] A. I. Karasin, K. West, S. Carman, and C. W. Olsen, "Characterization of avian $\mathrm{H} 3 \mathrm{~N} 3$ and $\mathrm{H} 1 \mathrm{~N} 1$ influenza A viruses isolated from pigs in Canada," Journal of Clinical Microbiology, vol. 42, no. 9, pp. 4349-4354, 2004.

[7] J. S. M. Peiris, Y. Guan, D. Markwell, P. Ghose, R. G. Webster, and K. F. Shortridge, "Cocirculation of avian H9N2 and contemporary "human" H3N2 influenza A viruses in pigs in southeastern China: potential for genetic reassortment?" Journal of Virology, vol. 75, no. 20, pp. 9679-9686, 2001.

[8] W. Ma, A. L. Vincent, M. R. Gramer et al., "Identification of H2N3 influenza A viruses from swine in the United States," Proceedings of the National Academy of Sciences of the United States of America, vol. 104, no. 52, pp. 20949-20954, 2007.

[9] D. Shoham, "The modes of evolutionary emergence of primal and late pandemic influenza virus strains from viral reservoir in animals: an interdisciplinary analysis," Influenza Research and Treatment, vol. 2011, Article ID 861792, 27 pages, 2011.

[10] S. Tong, Y. Li, P. Rivailler et al., "A distinct lineage of influenza A virus from bats," Proceedings of the National Academy of Sciences of the United States of America, vol. 109, pp. 42694274, 2012.

[11] Y. Kawaoka and R. G. Webster, "Sequence requirements for cleavage activation of influenza virus hemagglutinin expressed in mammalian cells," Proceedings of the National Academy of Sciences of the United States of America, vol. 85, no. 2, pp. 324328, 1988.

[12] H. Hatta, P. Gao, P. Halfmann, and Y. Kawaoka, "Molecular basis for high virulence of Hong Kong H5N1 influenza a viruses," Science, vol. 293, no. 5536, pp. 1840-1842, 2001.

[13] Z. Li, H. Chen, P. Jiao et al., "Molecular basis of replication of duck H5N1 influenza viruses in a mammalian mouse model," Journal of Virology, vol. 79, no. 18, pp. 12058-12064, 2005.
[14] Z. Li, Y. Jiang, P. Jiao et al., "The NS1 gene contributes to the virulence of $\mathrm{H} 5 \mathrm{~N} 1$ avian influenza viruses," Journal of Virology, vol. 80, no. 22, pp. 11115-11123, 2006.

[15] D. L. Noah, K. Y. Twu, and R. M. Krug, "Cellular antiviral responses against influenza $\mathrm{A}$ virus are countered at the posttranscriptional level by the viral NS1A protein via its binding to a cellular protein required for the $3^{\prime}$ end processing of cellular pre-mRNAS," Virology, vol. 307, no. 2, pp. 386-395, 2003.

[16] S. H. Seo, E. Hoffmann, and R. G. Webster, "Lethal H5N1 influenza viruses escape host anti-viral cytokine responses," Nature Medicine, vol. 8, no. 9, pp. 950-954, 2002.

[17] A. Jahangir, Y. Watanabe, O. Chinen et al., "Surveillance of avian influenza viruses in Northern pintails (Anas acuta) in Tohoku District, Japan," Avian Diseases, vol. 52, no. 1, pp. 4953, 2008.

[18] A. Jahangir, S. Ruenphet, S. Ueda et al., "Avian influenza and Newcastle disease viruses from northern pintail in Japan: isolation, characterization and inter-annual comparisons during 2006-2008," Virus Research, vol. 143, no. 1, pp. 44-52, 2009.

[19] A. Jahangir, S. Ruenphet, D. Shoham, M. Okamura, M. Nakamaura, and K. Takehara, "Haemagglutinin and neuraminidase characterization of low pathogenic $\mathrm{H} 5$ and $\mathrm{H} 7$ avian influenza viruses isolated from Northern pintails (Anas acuta) in Japan, with special reference to genomic and biogeographical aspects," Virus Genes, vol. 40, no. 1, pp. 94-105, 2010.

[20] E. Hoffmann, J. Stech, Y. Guan, R. G. Webster, and D. R. Perez, "Universal primer set for the full-length amplification of all influenza A viruses," Archives of Virology, vol. 146, no. 12, pp. 2275-2289, 2001.

[21] K. Tamura, J. Dudley, M. Nei, and S. Kumar, "MEGA4: Molecular Evolutionary Genetics Analysis (MEGA) software version 4.0," Molecular Biology and Evolution, vol. 24, no. 8, pp. 1596-1599, 2007.

[22] S. Liu, K. Ji, J. Chen et al., "Panorama phylogenetic diversity and distribution of type A influenza virus," PLoS ONE, vol. 4, no. 3, Article ID e5022, 2009.

[23] S. Ludwig, U. Schultz, J. Mandler, W. M. Fitch, and C. Scholtissek, "Phylogenetic relationship of the nonstructural (NS) genes of influenza A viruses," Virology, vol. 183, no. 2, pp. 566-577, 1991.

[24] S. Zohari, P. Gyarmati, P. Thorén et al., "Genetic characterization of the NS gene indicates co-circulation of two sublineages of highly pathogenic avian influenza virus of $\mathrm{H} 5 \mathrm{~N} 1$ subtype in Northern Europe in 2006," Virus Genes, vol. 36, no. 1, pp. 117-125, 2008.

[25] Y. Li, Z. Lin, J. Shi et al., "Detection of Hong Kong 97-like H5N1 influenza viruses from eggs of Vietnamese waterfowl," Archives of Virology, vol. 151, no. 8, pp. 1615-1624, 2006.

[26] A. Jahangir, S. Ruenphet, D. Shoham, M. Okamura, M. Nakamaura, and K. Takehara, "Phenotypic, genetic, and phylogeographical characterization of avian influenza virus subtype H5N2 isolated from northern pintail (Anas acuta) in Japan," Virus Research, vol. 145, no. 2, pp. 329-333, 2009.

[27] Y. Kawaoka, O. T. Gorman, T. Ito et al., "Influence of host species on the evolution of the nonstructural (NS) gene of influenza A viruses," Virus Research, vol. 55, no. 2, pp. 143-156, 1998.

[28] R. Chen and E. C. Holmes, "Avian influenza virus exhibits rapid evolutionary dynamics," Molecular Biology and Evolution, vol. 23, pp. 2336-2341, 2006.

[29] L. Duan, L. Campitelli, X. H. Fan et al., "Characterization of low-pathogenic H5 subtype influenza viruses from Eurasia: implications for the origin of highly pathogenic $\mathrm{H} 5 \mathrm{~N} 1$ 
viruses," Journal of Virology, vol. 81, no. 14, pp. 7529-7539, 2007.

[30] D. Shoham, "Biotic-abiotic mechanisms for long-term preservation and reemergence of influenza type A virus genes," Progress in Medical Virology, vol. 40, pp. 178-192, 1993.

[31] S. Zohari, P. Gyarmati, A. Ejdersund et al., "Phylogenetic analysis of the non-structural (NS) gene of influenza A viruses isolated from mallards in Northern Europe in 2005," Virology Journal, vol. 5, article 147, 2008.

[32] E. Spackman, D. E. Stallknecht, R. D. Slemons et al., "Phylogenetic analyses of type A influenza genes in natural reservoir species in North America reveals genetic variation," Virus Research, vol. 114, no. 1-2, pp. 89-100, 2005.

[33] Yamashina Institute for Ornithology, Japanese Bird Banding in Recent Years (1961-1985), Bird Migration Research Center, Abiko, Chiba, Japan, 1985.

[34] V. V. Bianki and I. N. Dobrynina, Migrations of Birds in Eastern Europe and Northern Asia, Nauka Press, Moscow, Russia, 1997.

[35] Yamashina Institute for Ornithology, Japanese Bird Banding in Recent Years (1961-2004), Bird Migration Research Center, Abiko, Chiba, Japan, 2004.

[36] C. A. Nicolai, P. L. Flint, and M. L. Wege, "Annual survival and site fidelity of northern pintails banded on the YukonKuskokwim Delta, Alaska," Journal of Wildlife Management, vol. 69, no. 3, pp. 1202-1210, 2005.

[37] M. R. Miller, J. Y. Takekawa, J. P. Fleskes, D. L. Orthmeyer, M. L. Casazza, and W. M. Perry, "Spring migration of Northern Pintails from California's Central Valley wintering area tracked with satellite telemetry: routes, timing, and destinations," Canadian Journal of Zoology, vol. 83, no. 10, pp. 1314-1332, 2005.

[38] J. Wahlgren, J. Waldenström, S. Sahlin et al., "Gene segment reassortment between American and asian lineages of avian influenza virus from waterfowl in the Beringia area," VectorBorne and Zoonotic Diseases, vol. 8, no. 6, pp. 783-790, 2008.

[39] L. Campitelli, A. Di Martino, D. Spagnolo et al., "Molecular analysis of avian H7 influenza viruses circulating in Eurasia in 1999-2005: detection of multiple reassortant virus genotypes," Journal of General Virology, vol. 89, no. 1, pp. 48-59, 2008.

[40] M. D. De Jong, T. T. Thanh, T. H. Khanh et al., "Oseltamivir resistance during treatment of influenza A (H5N1) infection," New England Journal of Medicine, vol. 353, no. 25, pp. 26672672, 2005.

[41] P. M. Colman, P. A. Hoyne, and M. C. Lawrence, "Sequence and structure alignment of paramyxovirus hemagglutininneuraminidase with influenza virus neuraminidase," Journal of Virology, vol. 67, no. 6, pp. 2972-2980, 1993.

[42] P. Ward, I. Small, J. Smith, P. Suter, and R. Dutkowski, "Oseltamivir (Tamiflu) and its potential for use in the event of an influenza pandemic," Journal of Antimicrobial Chemotherapy, vol. 55, no. 1, pp. i5-i21, 2005.

[43] Q. Zhu, H. Yang, W. Chen et al., "A naturally occurring deletion in its NS gene contributes to the attenuation of an H5N1 swine influenza virus in chickens," Journal of Virology, vol. 82, no. 1, pp. 220-228, 2008.

[44] J. C. Obenauar, J. Denson, P. K. Mehta et al., "Large-scale sequence analysis of avian influenza isolates," Science, vol. 311, no. 5767, pp. 1576-1580, 2006. 


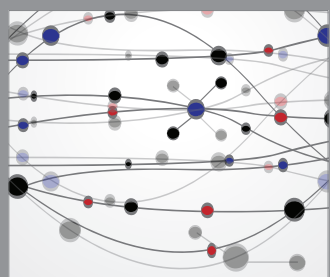

The Scientific World Journal
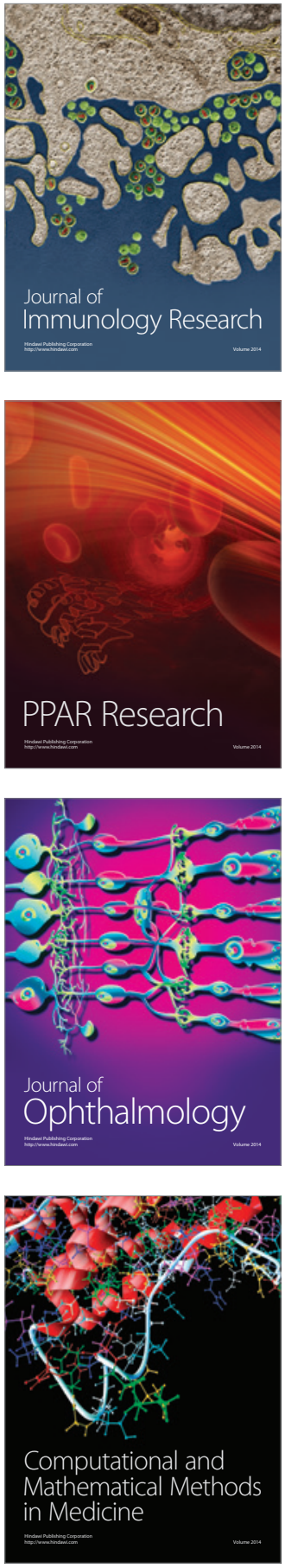

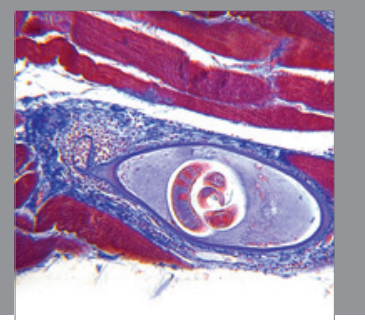

Gastroenterology

Research and Practice
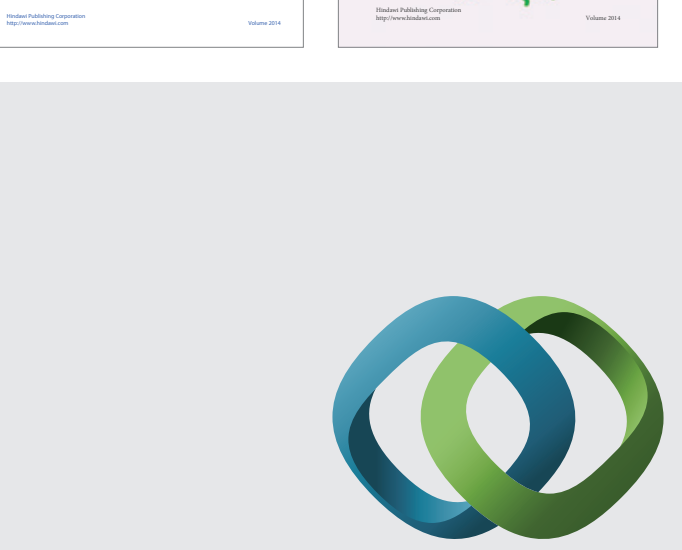

\section{Hindawi}

Submit your manuscripts at

http://www.hindawi.com
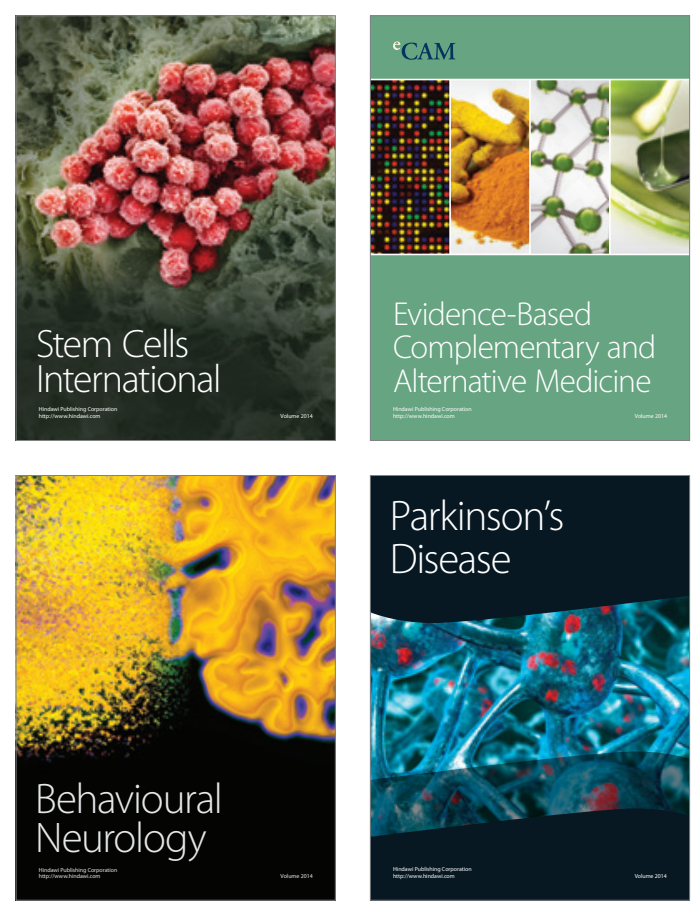

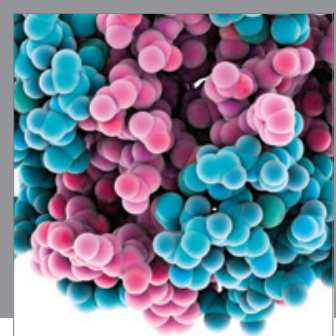

Journal of
Diabetes Research

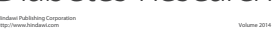

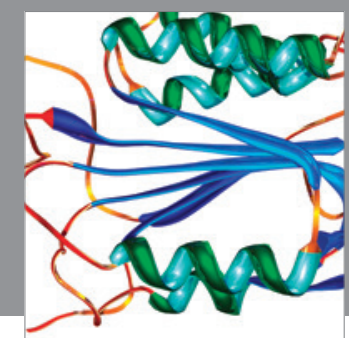

Disease Markers
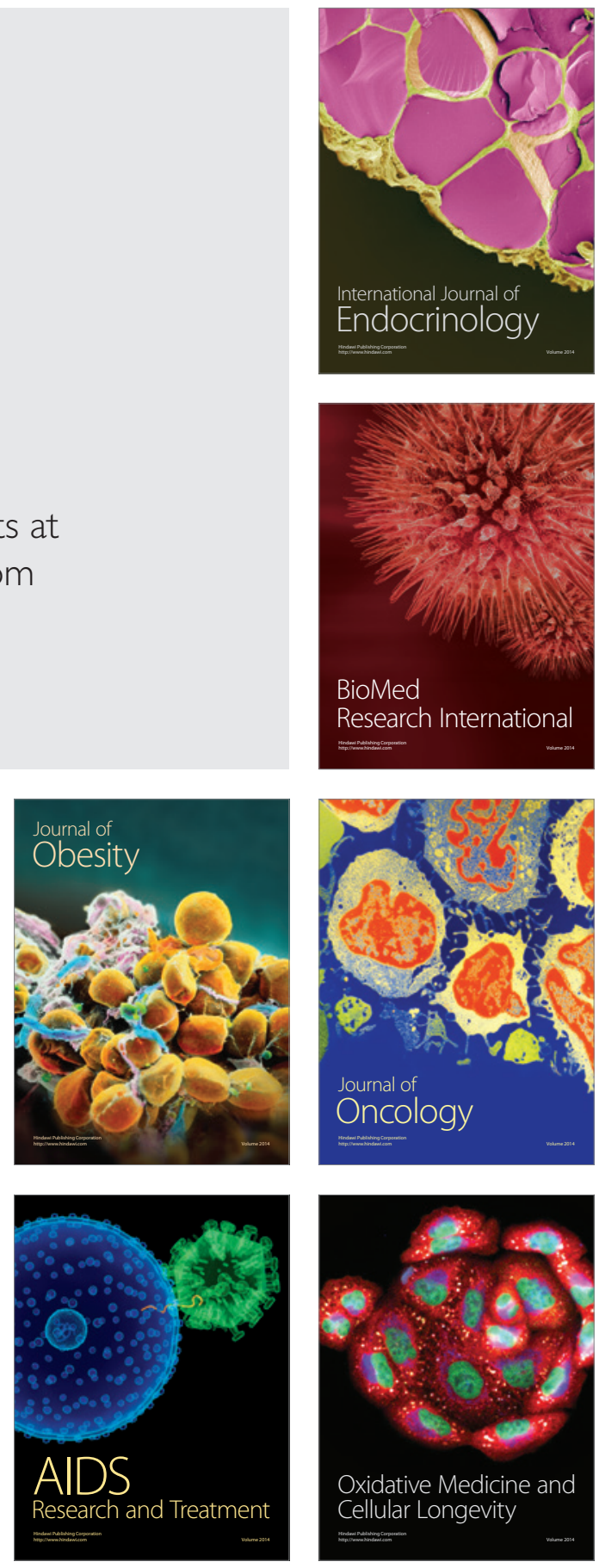